\title{
The Analysis on the Relationship between Dating and the Gel-ink Material Penetration of Handwriting along Z Direction on Paper
}

\author{
Jiangchun LI ${ }^{1}$, Yuxiao YANG ${ }^{2}$, Chen Wang ${ }^{2}$, Xiaobo XIONG ${ }^{1^{*}}$ \\ ${ }^{I}$ The key Laboratory of Forensic science in Hubei province, Hubei university of Police, Wuhan, Hubei, \\ China \\ ${ }^{2}$ Engineering Research Center of Nano-Geo Materials of Ministry of Education of China University of \\ Geosciences, Wuhan, Hubei, China \\ *corresponding author.
}

\begin{abstract}
Scanning electron microscopy (SEM) technology has been widely used in forensic science, which promotes the development of interdisciplinary science. This paper used SEM to observe the penetration degree of common black gel-ink on paper. The penetration morphology of the different black brands gel-ink has been observed. The relationship between the penetration process of gel-ink material and the dating of document has been observed after determining the measuring position. The results showed that the penetration depth of ink along $Z$ direction on paper is significantly different, the penetration speed of ink is also different, which presents regular variety and gradually reaches a relatively stable state over time. The application of SEM will provide a useful exploration for judging the ink dating.
\end{abstract}

Keywords: SEM, black gel-ink, material, penetration, ink dating

\section{Introduction}

With the development of science and technology, there are more and more difficult problems in the practice of judicial document identification [1], The number of cases involving the identification of the dating of suspicious document evidence is increasing year by year, and the identification of the dating of document evidence ink even becomes the only basis for identifying the authenticity of documents. Therefore, Forensic scientists pay more attention to the identification of the dating when documents are made in forensic expertise. Due to the practical difficulties such as numerous and complex writing pigments, lack of effective inspection technology and limited application scope of existing technology, it has become an inevitable trend to speed up the research on the inspection technology of dating of document $[2,3]$.

Once the writing ink forms on the paper-based carrier, there will be diffusion, penetration and other phenomena. In addition to the horizontal diffusion / diffusion along the fiber in the $\mathrm{X}$ and $\mathrm{Y}$ axis of the horizontal plane, it will also penetrate into the paper along the fiber and pore in the $\mathrm{Z}$ direction. Modern testing and analysis technology has been skilfully used in the field of judicial expertise. Researchers at home and abroad have done a lot of fruitful research work and achieved rich results, such as Raman spectroscopy [4, 5], ultraviolet visible absorption spectroscopy [6,7], X-ray fluorescence spectroscopy [8,9], scanning electron microscopy [10, 11], etc., which are widely used in the field of judicial expertise the species of the physical evidence such as stamp oil and paper money can be effectively detected, analysed and identified. It can be seen from the literature retrieval that the research on ink penetration mainly involves in the printing field, focusing on the influence of ink penetration in paper on printing quality and effect, so as to avoid the problems of dot enlargement, long drying time, dirt rubbing or penetration [12]; The measurement methods of penetration depth of ink in paper include direct measurement by cutting, such as adding traceable substances into ink or using electron microscope technology, and quantitative measurement by measuring the difference of light reflectivity between printed paper and ink. With the improvement and progress of micro technology, although the paper needs to be cut, it can obtain higher ISSN: 0010-8189 
measurement accuracy. This method is still desirable in the study of general paper penetration problems [13].

It is generally believed that ink penetration can be divided into free penetration and pressure penetration. Free penetration mainly depends on the viscosity of ink carrier, the absorption properties of binder (adhesive) and substrate. Pressure penetration is not only related to paper porosity and ink characteristics, but also related to printing speed, paper and printing pressure [14]. In order to facilitate the discussion on the penetration of ink and paper, Bristow established a mathematical model to calculate the penetration depth of ink based on the propagation of light in uniform medium according to K-M theory [15]. Li Yingying et al. Summed up the shortcomings of the model, revised the K-M theory, and studied the diffusion and penetration of ink in the paper more comprehensively and accurately [16], or assumed that the capillary tubes are parallel arranged pores perpendicular to the paper surface, and used this pore system as the paper model to analyse [17]. In the field of forensic science, the diffusion degree of ink is studied, in which the diffusion degree of ink or printing ink is determined by the distance of sulfate ion in blue black ink on the paper surface with time diffusion, which is one of the very effective methods to determine the dating of handwriting [18]. But this method also has its own limitations: first, the limitations of the detection object, it is only limited to the detection of blue and black ink; Second, the detection dimension only involves the measurement of the ink diffusion distance in the horizontal $\mathrm{X}$ and $\mathrm{Y}$ axis direction, and does not study the ink penetration depth in the vertical $\mathrm{Z}$ direction $[19,20]$.

Therefore, we can see that although there is a more in-depth study on ink and paper penetration, there is no relevant research on the relationship between ink penetration and time in the field of forensic expertise. In view of the above shortcomings and limitations, this study is based on the penetration problem of writing ink, using electronic scanning microscopy technology to explore the penetration law of writing ink on paper, research and detect the relationship and model of writing ink on the $\mathrm{Z}$ dimension of paper with time, so as to break through the limitation of only detecting blue and black ink and broaden the range of ink types, And it can provide a new detection method and means for the discrimination of ink formation time, and provide a certain theoretical basis for the discrimination of forensic document formation time.

\section{Manuscript Preparation}

\subsection{Reagents and instruments}

The field emission scanning electron microscope (FESEM), SU8010, Hitachi, Japan produced by Hitachi, Japan, has an accelerating voltage of $20 \mathrm{kV}$.

\subsection{Experimental process}

Sample preparation: six brands of black neutral pens were collected in Wuhan, with the nib diameter of $0.5 \mathrm{~mm}$ and numbered respectively (see Table 1); Draw straight lines on $70 \mathrm{~g} / \mathrm{m} 2$ copy paper, $75 \mathrm{~g} / \mathrm{m} 2$ copy paper and coated paper to form samples. The prepared samples were put into a vacuum dryer overnight to remove moisture. Then observe the sample with a certain voltage. In order to prevent A4 printing paper from burning, low voltage operation should be preferred. In turn, use scissors to cut a square piece of paper about $1 \mathrm{~cm} 2$, and select the ink color as consistent as possible; the ink was cut in two halves vertically along the direction of ink stroke. The ink was pasted on the slide with double-sided adhesive tape and placed on the sample table. The sample table was kept in the SEM vacuum chamber. Each sample was analyzed under different operating parameters (such as magnification, working distance, source electron energy and current, etc.) to observe the ink penetration morphology, the image is captured and saved in JPEG format.

In the first observation, the characteristic points visible in the field of vision of the sample under the same scanning scale should be determined first, so as to ensure the same analysis area when the subsequent characterization is repeated, and the dynamic rules of the change of the cross region near the feature points are summarized to ensure

ISSN: 0010-8189 
the universality of the experiment. Follow the above steps to perform the same operation on the black ink No. 2-6.

Storage conditions: laboratory temperature $\left.\left(20^{\circ} \mathrm{C}\right) \pm 5^{\circ} \mathrm{C}\right)$, relative humidity $(\mathrm{RH})$ is $40-60 \%$ and stored naturally.

Table 1 list of six common brands of Black Signature pens

\begin{tabular}{cccc}
\hline Nu & Brand & Model & Place of Origin \\
\hline 1 & Deli & S656 & Deli group, China \\
2 & ZEBRA & Z-Grip & Zebra group, Japan \\
3 & M\&G & AGR640S9 & Chenguang company, China \\
4 & Pentel & BLA75C2-A & Paycom, Japan \\
5 & Schneider & 861 & Schneider, Germany \\
6 & Pilot & B2P & Baile, Japan \\
\hline
\end{tabular}

2.3 testing and characterization

Observe the handwriting samples written on the copy paper, take photos of the ink penetration section, and record the measured values. The time of taking photos and recording is the formation of fresh writing, the 3rd day, the 5th day and the 15th day, respectively, to observe the penetration law. For each sample material, fixed position points are selected and measured to obtain the distance value of ink penetration depth after the measured value. The first measured value is recorded as the original ink penetration depth, which is represented by $\mathrm{L}_{1}$; the distance values of each position point are measured in different time periods and recorded $\operatorname{asL}_{2}, \mathrm{~L}_{3}$, $\mathrm{L} 4$ etc. Therefore, the relative penetration depth $\triangle D_{1}$ relative $=L_{2}-L_{1}$ at the first measurement can be obtained, and $\triangle D_{2}, \triangle D_{3}$ etc. can be obtained in turn. Then the data were analyzed and compared.

Rate formula $\mathrm{V}=\mathrm{S} / \mathrm{T}$ (where $\mathrm{V}$ is ink penetration rate, $\mathrm{S}$ is ink penetration distance and $\mathrm{T}$ is ink penetration time)

\section{Results and discussion}

3.1 Determination of measurement position

In fact, the paper is a kind of porous structure, capillary distribution is very complex, pore size is different, arranged crisscross, when the writing ink on the paper, the ink will gradually penetrate into the paper with time, there is an obvious boundary in the cross-section of paper and ink penetration. The ink penetration depth at different positions is uneven, and the ink penetration boundary is also uneven. The surface is uneven due to fiber interweaving observed by SEM. The data obtained at different ink penetration positions are quite different, and the measurement error is large (see Fig. 1a). If we use a prominent marker, such as a prominent and easily recognized fiber point, as the measurement position, it is not only easy to quickly identify the measurement position, but also has small measurement error (see Fig. 1b). Therefore, we use the method of marking the protrusion to determine the measurement position of the ink, and then through the demarcation and calculation of the boundary of the penetration surface, we can obtain the relative penetration value of the ink ( $\triangle \mathrm{D}$ relative). 

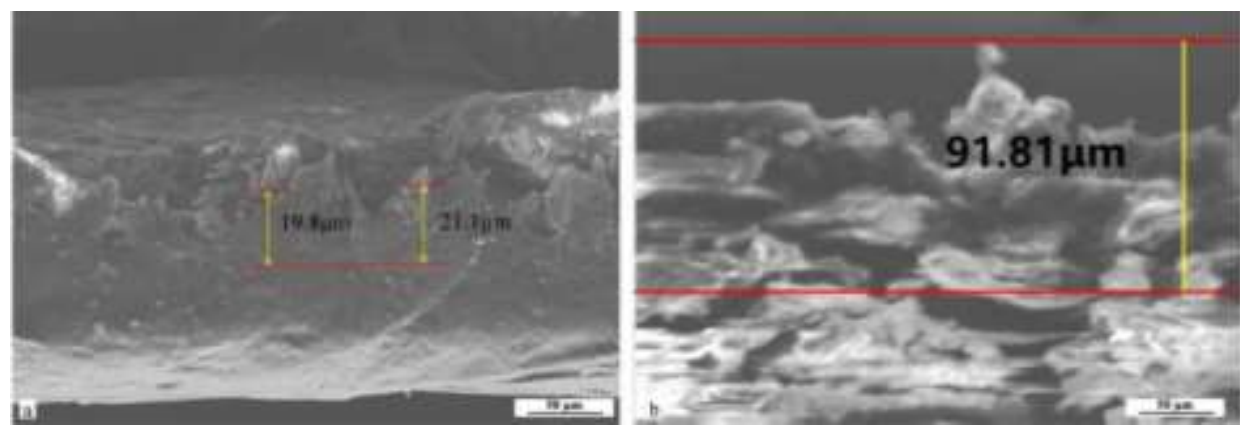

Fig. 1 SEM image of ink penetration value when different measuring positions are selected, where a is the measurement value of no obvious mark position; $B$ is the measurement value with obvious mark.

\section{2 variation of the penetration depth of ink cross section with time}

The cross-section of the written sample was analyzed by FESEM. It can be seen clearly that the paper structure of this part has regular changes. According to the experiment, the cross-section ink penetration depth and the relative penetration depth within the time interval (see Table 2 and table 3) can be seen that all the ink of the sample handwriting will gradually penetrate into the paper with the time (see Fig. 2), and then reach a relatively stable state. It can be seen that the permeability depth value of the second $\triangle \mathrm{D} 2$ of $\mathrm{S} 1$ and $\mathrm{S} 3$ sample sections shown in Fig. 1 is much larger than that of S2, S4, S5 and S6 samples; Except S2 and S6, the third $\triangle \mathrm{D} 3$ of other samples has a relatively small increase in permeability depth (see figures 3 and 4). The penetration depth of different paper and ink is different, which is affected by the paper coating structure and other factors.

According to the rate formula, the penetration rates of six samples in different time periods can be calculated (see Table 2). It can be observed that under the same storage conditions, the penetration rates of different brands of ink are quite different; on the fifth day, the penetration rate decreased rapidly and began to narrow the gap gradually. On the 15th day, the penetration rate was less than $9 \mathrm{~m} / \mathrm{day}$. It can be seen from Figure 5 that the ink penetration rate is the highest on the first day, the fastest decline is found on the third and fifth day, and the decline is gradually gentle on the 15th day.
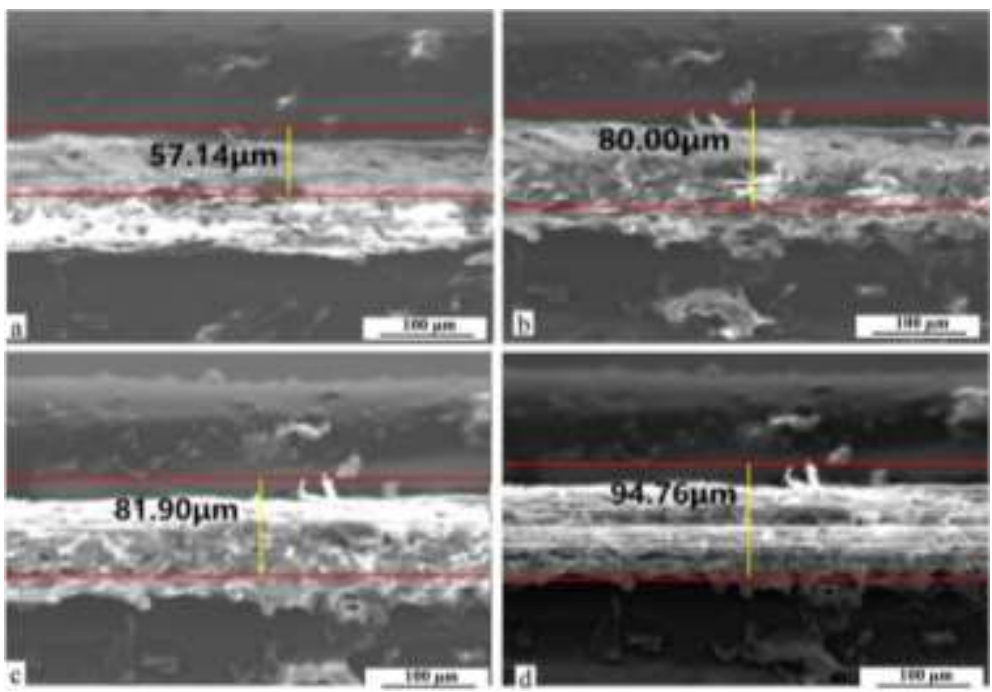

Fig. 2 SEM image of the penetration depth of zebra Z-Gri black signature pen with time,

Among them, a was fresh writing, $b$ was measured after 3 days, $c$ was measured after 5 days, and $d$ was measured after 15 days

Table 2 penetration depth and penetration rate of ink in sample paper section with different time

ISSN: 0010-8189

(C) CONVERTER 2020

www.converter-magazine.info 
CONVERTER MAGAZINE

Volume 2021, No. 4

\begin{tabular}{|c|c|c|c|c|c|c|c|c|}
\hline \multirow{2}{*}{$\begin{array}{l}\text { Nu/time } \\
\text { interval }\end{array}$} & \multicolumn{2}{|c|}{1 day } & \multicolumn{2}{|c|}{3 day } & \multicolumn{2}{|c|}{5 day } & \multicolumn{2}{|c|}{15 day } \\
\hline & $\begin{array}{c}\mathrm{L} 1 \\
(\mu \mathrm{m})\end{array}$ & $\begin{array}{l}\mathrm{V} 1 \\
\mu \mathrm{m} / \text { day })\end{array}$ & $\begin{array}{c}\mathrm{L} 2 \\
(\mu \mathrm{m})\end{array}$ & $\begin{array}{c}\mathrm{V} 2(\mu \mathrm{m} / \mathrm{day} \\
)\end{array}$ & $\begin{array}{c}\text { L3 } \\
(\mu \mathrm{m})\end{array}$ & $\begin{array}{c}\mathrm{V} 3(\mu \mathrm{m} / \mathrm{day} \\
)\end{array}$ & $\begin{array}{c}\mathrm{L} 4 \\
(\mu \mathrm{m})\end{array}$ & $\begin{array}{c}\mathrm{V} 4(\mu \mathrm{m} / \mathrm{day} \\
)\end{array}$ \\
\hline $\mathrm{S} 1$ & 110.00 & 110.00 & 113.81 & 37.94 & 124.76 & 24.95 & 128.09 & 8.54 \\
\hline $\mathrm{S} 2$ & 57.14 & 57.14 & 80.00 & 26.67 & 81.90 & 16.38 & 94.76 & 6.32 \\
\hline $\mathrm{S} 3$ & 101.90 & 101.90 & 105.24 & 35.08 & 116.67 & 23.33 & 118.09 & 7.87 \\
\hline $\mathrm{S} 4$ & 67.62 & 67.62 & 85.71 & 28.57 & 91.90 & 18.38 & 97.61 & 6.51 \\
\hline S5 & 35.24 & 35.24 & 50.00 & 16.67 & 62.86 & 12.57 & 64.76 & 4.32 \\
\hline S6 & 74.76 & 74.76 & 88.57 & 29.52 & 90.95 & 18.19 & 100.95 & 6.73 \\
\hline
\end{tabular}

Table 3 relative penetration depth of ink in sample paper section with different time/ $\mu \mathrm{m}$

\begin{tabular}{llll}
\hline $\mathrm{Nu}$ & $\triangle \mathrm{D} 1$ relative & $\triangle \mathrm{D} 2$ relative & $\Delta \mathrm{D} 3$ relative \\
\hline $\mathrm{S} 1$ & 3.81 & 14.76 & 18.09 \\
$\mathrm{~S} 2$ & 22.86 & 24.76 & 37.16 \\
$\mathrm{~S} 3$ & 3.34 & 14.77 & 16.19 \\
$\mathrm{~S} 4$ & 18.09 & 24.28 & 29.99 \\
$\mathrm{~S} 5$ & 14.76 & 27.62 & 29.52 \\
$\mathrm{~S} 6$ & 13.81 & 16.19 & 26.19 \\
\hline
\end{tabular}

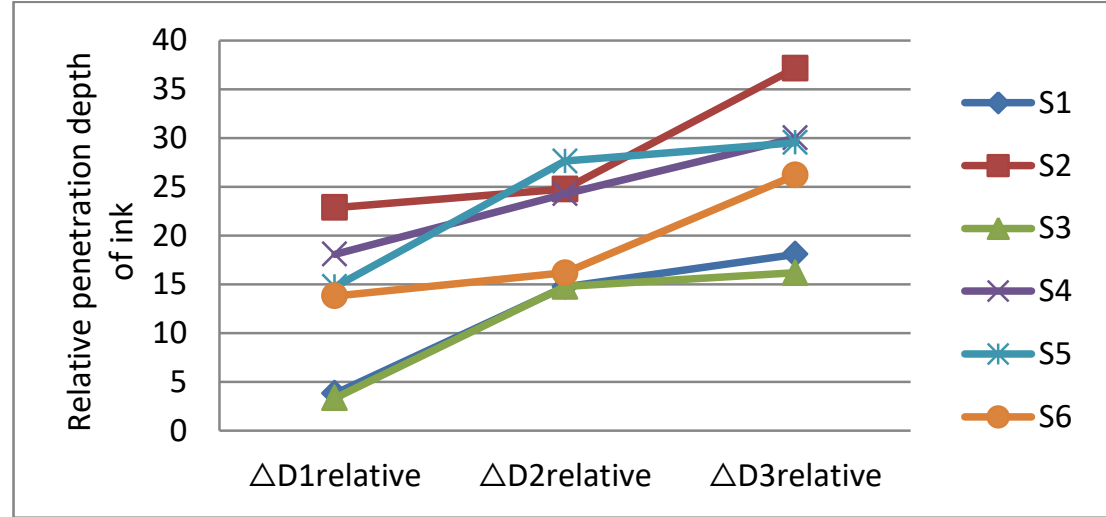

Fig. 4 Relation between relative penetration depth and time

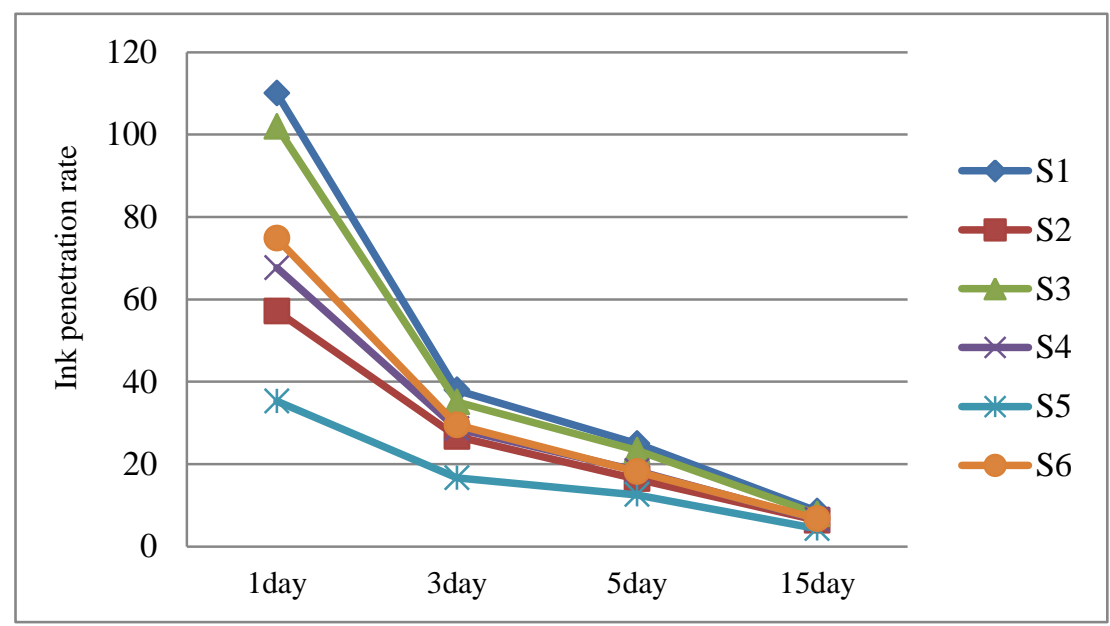

ISSN: 0010-8189 
3.3 Influence of different paper on ink penetration depth

Under the same storage and production conditions, six different brands of ink were written on three different kinds of paper $(70 \mathrm{~g} / \mathrm{m} 2$ copy paper, $75 \mathrm{~g} / \mathrm{m} 2$ copy paper and coated paper respectively). The characterization results of electron microscope are shown in Figure 6. Taking the penetration section observation of pilot B2P black neutral pen sample ink as an example, we can see that the ink forms a uniform penetration surface in the sample section, and there is an obvious dividing line in the section topography of the three samples. The relative penetration depth of pilotb2p brand black neutral pen ink on $75 \mathrm{~g} / \mathrm{m} 2$ copy paper is the largest and the fastest (Fig. 6b), while the relative penetration depth on $70 \mathrm{~g} / \mathrm{m} 2$ copy paper and coated paper is less than $75 \mathrm{~g} / \mathrm{m} 2$, and the penetration depth on coated paper is the slowest (Fig. 6a, FIG. 6c), reflecting that different paper types have obvious effects on the penetration depth of ink, The penetration rate of ink has a great influence on the fiber tightness and sizing degree of paper, but it does not affect the penetration into the paper with time.

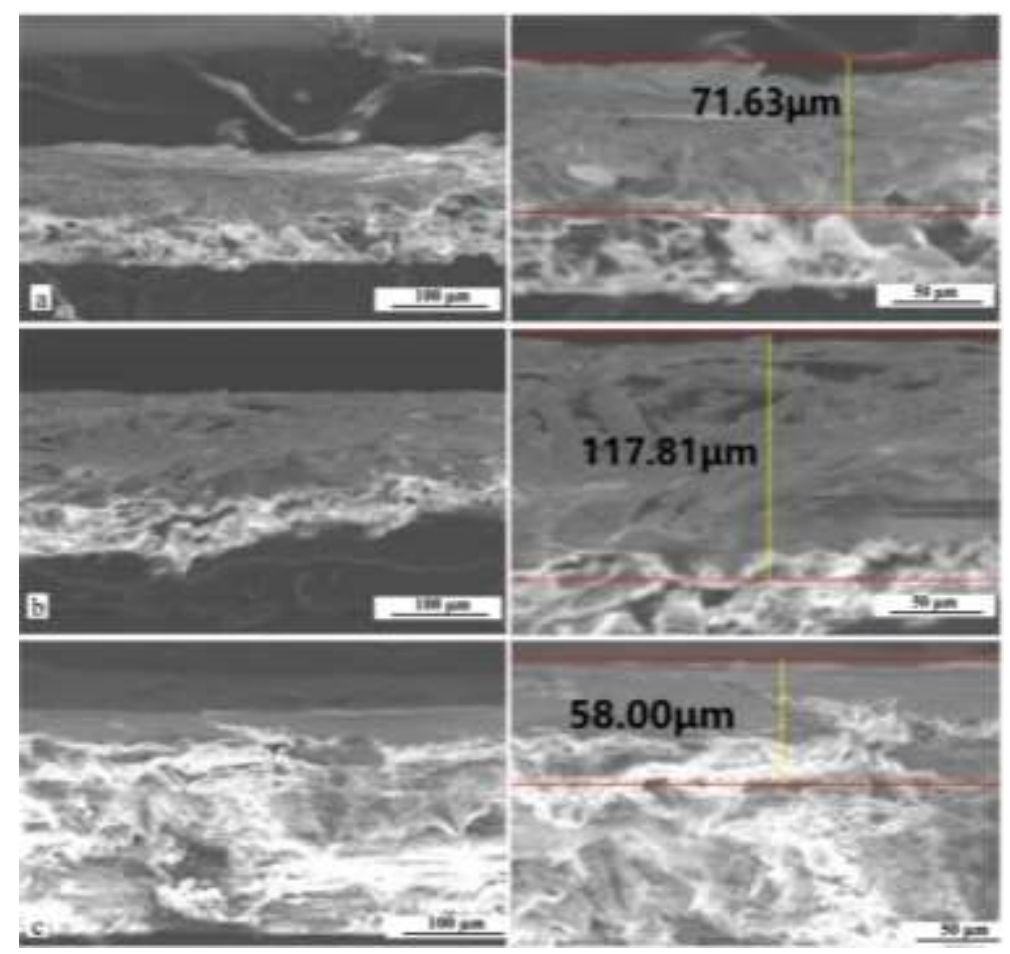

Fig. 6 SEM images of ink penetration section of pilot B2P black neutral pen samples on different papers. Bar $=100$ on the left $\mu \mathrm{m}$. Right bar $=50 \mu \mathrm{m}$ a is $70 \mathrm{~g} / \mathrm{m} 2$ copy paper; $b$ is $75 \mathrm{~g} / \mathrm{m} 2$ copy paper; $c$ is coated paper

\section{Conclusion}

In this paper, through the measurement of penetration depth of several common black neutral pen inks, it is found that there is an obvious interface between light and dark in the cross-section of ink penetration, which can be used to measure the penetration depth of ink more accurately. At the same time, it is found that the penetration depth and penetration speed of ink on different papers are different. With the passage of time, the penetration speed of ink is first fast and then slow, and gradually reaches a relatively stable state, which provides a theoretical basis for establishing the relationship between the penetration depth and the formation time of ink by using electronic scanning microscopy, it provides a new idea for the inspection of dating of documents.

At the same time, we should also see that ink penetration is a complex process, and many problems have not been

ISSN: 0010-8189 
solved. Due to time, experimental conditions, sample collection and other factors, there are still deficiencies in research depth and application. We need to continue to observe and improve the critical point of ink penetration.

\section{Acknowledgements}

This work was sponsored by Natural Science Foundation of Hubei Province in 2019 (2019CFB747) and Hubei Key Laboratory of Forensic Science (KFKT2019001).

\section{References}

[1] Y. Zhang, J. Yu, "Research on the application of modern analytical technology in document inspection. 25th annual meeting of Chinese Chemical Society--Proceedings of public safety chemistry branch," Beijing Institute of criminal science and technology, pp. 52-55, 2006.

[2] Y. X. Gan, F. Yang, J. Wu, Y. Yu, “Application of scanning electron microscope in rock and mineral analysis," Journal of electron microscopy, vol. 38, no. 03, pp. 284-293, 2019.

[3] L. X. Wang, Q. M. Xu, "Identification and application of scanning electron microscopy to the ashes of common textiles," Journal of electron microscopy, vol. 38, no. 04, pp. 397-402, 2019.

[4] L. Wu, Z. H. Ouyang, S. H. Cao, et al., "Application and research progress of Raman spectroscopy," Journal of light scattering, vol. 17, no. 2, pp. 180-186, 2005.

[5] P. C. WHITE, "SERRS spectroscopy, a new technique for forensic science?" Science \& Justice, vol. 40, no. 2, pp.113-119, 2000.

[6] X. F. Guo, "Identification of the types of printing oil by UV-vis spectroscopy," Guangdong Public Security Science and technology, vol. 25, no. 02, pp. 18-22, 2017.

[7] S. H. Zhuo, "X-ray fluorescence spectroscopic analysis," Laboratory, vol. 28, no. 07, pp.112-122, 2009.

[8] S. L. Hu, H. Shen, R. David, et al., "Application of micro beam X-ray fluorescence spectrometry in the examination of physical evidence of a black paper," Journal of analytical and testing, vol. 28 no.7, pp. 824-828. 2009.

[9] D. K. WILLIAMS, A. A. VITAL, "Study to investigate the evidential value of blue gel pen inks," Journal of Forensic Sciences, vol. 48, no. 2, pp. 419-424, 2003.

[10] T. T. Nie, "Study on various testing methods of black signature pen ink," Shenyang: Liaoning Normal University, 2006

[11] F. P. Liu, X.K. Qi, A.L. Wang, "Calculation and experimental study of ink penetration depth in printing process," Packaging Engineering, no. 01, pp. 71-75, 2006.

[12] R. A. Li, "Research on printability and evaluation method of uncoated inkjet paper," Master's Thesis of Nanjing Forestry University, no. 6, pp. 2-11.

[13] F. P. Liu, X. K. Qi, A. L. Wang, "Research methods of ink penetration in paper," Packaging Engineering, no. 05, pp. 13-15, 2006.

[14] J. A. Bristow, L. Zhang, "Mathematical expression of permeating printing and ink permeating," Wu Test Translation, no. 4, pp. 38-42, 1992.

[15] Y.Y. Liu, J. H. Qian, "Model study on the influence of ink transfer penetration on printing quality," Packaging Engineering, vol. 33, no. 03, PP. 117-120.

[16] G. S. Hu, C. X. Zhang, Y. H. Ma, et al., "Study on capillary distribution and ink penetration of paper," Journal of Wuhan University (Information Science Edition), no. (09), pp. 829-831, 2006.

[17] D. Y. Han, L. Y. Tu, "Document inspection," Beijing: China People's Public Security University Press," pp. 242-245, 2015.

[18] A. Koenig, J. B ü gler, D. Kirsch, et al., "Ink dating using thermal desorption and gas chromatography/mass spectrometry: comparison of results obtained in two laboratories," Journal of Forensic Sciences, vol. 60, no. s1, pp. s152-s161, 2014.

[19] M. Suzuki, N. Akiba, K. Kurosawa, et al., "Differentiation of black writing ink on paper using luminescence lifetime by time-resolved luminescence spectroscopy," Forensic Science International, vol. 279, pp. 281-287, 2017.

ISSN: 0010-8189 